\title{
Alterations of thyroid microbiota across different thyroid microhabitats in patients with thyroid carcinoma
}

Daofeng Dai ${ }^{1+}$, Yan Yang ${ }^{2+}$, Yong Yang ${ }^{3+} \mathbb{D}$, Tianfeng Dang ${ }^{1}$, Jiansheng Xiao ${ }^{4}$, Weibin Wang ${ }^{2}$, Lisong Teng ${ }^{2 *}$, Juan $\mathrm{Xu}^{5^{*}}$, Jing $\mathrm{Ye}^{1^{*}}$ and Hongqun Jiang ${ }^{1^{*}}$

\begin{abstract}
Background: In recent years, the incidence rate of Thyroid carcinoma (TC) has been increasing worldwide. Thus, research on factors of TC carcinogenesis may promote TC prevention and decrease the incidence rate. There are several studies targeting the correlation between gut microbiota and thyroid disease. Carcinogenesis of several malignancies is influenced by microbiota. However, thyroid microbiome of TC has not been revealed. This study investigated thyroid microbiota in different TC microhabitats.
\end{abstract}

Methods: We performed 16s rRNA gene sequencing using tumor tissues and matched peritumor tissues from 30 patients with TC to characterize thyroid microbiota.

Results: The richness and diversity of thyroid microbiota were lower in TC tumor samples than in matched peritumor tissues. At the genus level, the core microbiota of thyroid included Sphingomonas, Comamonas, Acinetobacter, Pseudomonas, Microvirgula, and Soonwooa. The abundance of Sphingomonas and Aeromonas was significantly increased in tumor tissues, while the abundance of Comamonas, Acinetobacter, and Peptostreptococcus was significantly enhanced in peritumor tissues. The combination of Comamonas and Sphingomonas could discriminate tumor samples from peritumor samples with an area under the curve (AUC) of 0.981 (95\% confidence interval [CI] 0.949-1.000). The abundance of Sphingomonas was significantly higher in N1 stage than in N0 stage. Sphingomonas could distinguish between N0 and N1 stage with an AUC of 0.964 (95\% Cl 0.907-1.000).

Conclusions: The microbial diversity and composition were significantly different between peritumor and tumor microhabitats from patients with TC, which may eventually affect TC carcinogenesis and progression. The combination of Comamonas and Sphingomonas could serve as a powerful biomarker for discrimination between tumor and peritumor tissues. Furthermore, the higher abundance of Sphingomonas was correlated with lymph node metastasis,

\footnotetext{
*Correspondence: Isteng@zju.edu.cn; hillyxj@sina.com;

yjholly@email.ncu.edu.cn; jianghq@ncu.edu.cn

${ }^{\dagger}$ Daofeng Dai, Yan Yang and Yong Yang contributed equally to this article

1 Jiangxi Otorhinolaryngology Head and Neck Surgery Institute,

Department of Otorhinolaryngology Head and Neck Surgery, The First

Affiliated Hospital of Nanchang University, Nanchang, China

${ }^{2}$ Department of Surgical Oncology, The First Affiliated Hospital, School

of Medicine, Zhejiang University, Hangzhou, China

${ }^{5}$ Pathology Department, Taizhou Hospital of Zhejiang Province Affiliated

to Wenzhou Medical University, Linhai, China

Full list of author information is available at the end of the article
}

(c) The Author(s) 2021. Open Access This article is licensed under a Creative Commons Attribution 4.0 International License, which permits use, sharing, adaptation, distribution and reproduction in any medium or format, as long as you give appropriate credit to the original author(s) and the source, provide a link to the Creative Commons licence, and indicate if changes were made. The images or other third party material in this article are included in the article's Creative Commons licence, unless indicated otherwise in a credit line to the material. If material is not included in the article's Creative Commons licence and your intended use is not permitted by statutory regulation or exceeds the permitted use, you will need to obtain permission directly from the copyright holder. To view a copy of this licence, visit http://creativecommons.org/licenses/by/4.0/. The Creative Commons Public Domain Dedication waiver (http://creativeco mmons.org/publicdomain/zero/1.0/) applies to the data made available in this article, unless otherwise stated in a credit line to the data. 
indicating that the abundance of Sphingomonas may indicate a poor prognosis for TC patients, and Sphingomonas may play a role in promoting TC progression.

Keywords: Thyroid cancer, Microbiome, Lymph node metastasis, Biomarker, Sphingomonas

\section{Introduction}

Thyroid carcinoma (TC) is a common endocrine malignancy, with an estimated 567,000 new cancer cases and 41,000 deaths worldwide in 2018 [1]. The main histological types include papillary thyroid carcinoma (PTC), follicular thyroid carcinoma (FTC), Hurthle cell thyroid carcinoma (HCTC), medullary thyroid carcinoma (MTC), and anaplastic thyroid carcinoma (ATC), which account for $80.2 \%, 11.4 \%, 3.1 \%, 3.5 \%$, and $1.7 \%$ of thyroid cancer, respectively [2]. In recent years, the incidence rate of TC has been increasing worldwide. Thus, research on factors of TC carcinogenesis may promote TC prevention and decrease the incidence rate.

Several organs, such as lungs, bladder, and urethra, have long been considered sterile. The advent of nextgeneration sequencing reveals that these organs are inhabited by a robust microbiota $[3,4]$. Due to the acidic environment of the human stomach, researchers previously believed that the stomach was not suitable for the growth of other microorganisms and was inhabited exclusively by Helicobacter pylori. However, recent advances in sequencing technology make it clear that the stomach is colonized by a huge number of microorganisms [5]. Dysbiosis of gastric microbiota can affect metabolism, inflammation, immunity [6,7], and eventually result in gastric cancer [8]. The imbalance between the types of microorganisms within the lung can cause lung diseases, such as cystic fibrosis [9], asthma [10], chronic obstructive pulmonary diseases [11], or even lung cancer [12]. However, the profile and functional role of thyroid microbiome in patients with TC has not been revealed.

There are several studies targeting the correlation between gut microbiota and thyroid disease. A study reported that the proportions of Pasteurellaceae and Prevotellaceae were higher, but the proportions of Veillonellaceae, Enterobacteriaceae, and Rikenellaceae were significantly lower in patients with Graves' disease compared to controls [13]. The abundance of Lactobacillaceae and Bifidobacteria was reduced, but the abundance of Enterococcus spp. was increased in hyperthyroid patients compared to healthy controls [14]. Su et al. reported that they observed significant differences in alpha and beta diversities of gut microbiota between patients with primary hypothyroidism and healthy individuals [15]. The fecal microbiota transplantation showed that total thyroxine levels were decreased in mice receiving the transplant from patients with primary hypothyroidism. Gut microbiota diversity and composition were significantly different between patients with $\mathrm{TC}$ and healthy controls [16].

The term 'thyrogastric syndrome' referring to the link between the gastrointestinal tract and the thyroid has been postulated in 1950s [17]. Gastric mucosal cells and thyroid follicular cells have the same embryonic origin because the thyroid gland develops from primitive gut cells [18]. Thus, we hypothesize that thyroid gland may also be colonized by microorganisms. For the first time, we performed 16s rRNA gene sequencing using tumor tissues and matched peritumor tissues from 30 patients with TC to characterize the core microbiota of thyroid, compare microbial diversity and composition of tumor tissues and matched peritumor tissues, identify differential taxa between tumor tissues and matched peritumor tissues, and characterize the microbial biomarkers for discrimination between tumor and peritumor tissues. The correlation between microbiota of thyroid carcinoma and clinicopathological factors was analyzed.

\section{Methods}

This retrospective study included 55 TC patients who underwent total thyroidectomy between March 2018 and December 2018 at the First Affiliated Hospital, School of Medicine, Zhejiang University. Two patients with body mass index $(\mathrm{BMI})>30$, two patients with history of malignancy or receiving radiotherapy/ chemotherapy before operation, ten patients with recent usage of antibiotics, probiotics, prebiotics, symbiotics, and eleven patients with no paired tissues were excluded (Fig. 1). Finally, 30 subjects were obtained for analysis of thyroid microbiota. The tumor

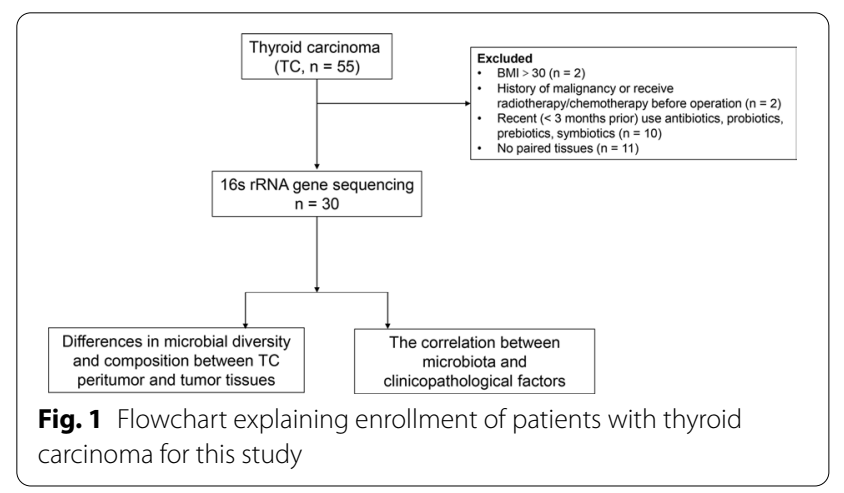


and peritumor (about $3 \mathrm{~cm}$ adjacent to the cancer tissue) tissues were collected, which were confirmed by pathological diagnosis. Archival slides of patients were evaluated by two pathologists. We obtained the following clinicopathological information: gender, age upon diagnosis, tumor size, extrathyroidal extension, recurrence risk stratification, and clinical stage. TNM staging was determined based on the 8th edition of the American Joint Committee on Cancer staging system. The clinicopathological information was supplied in Table 1. Approval for this study was obtained from Medical Ethics Committee of the First Affiliated Hospital, School of Medicine, Zhejiang University. Patients signed an informed consent.
DNA extraction, amplicon library construction, and sequencing

The genomic DNA of thyroid tissues (about $100 \mathrm{mg}$ ) was extracted using cetyltrimethylammonium bromide/ sodium dodecyl sulfate method. To evaluate environmental contamination, six sterile Petri dishes with sterile water and sterile filter paper were placed in different corners of the operating room for $24 \mathrm{~h}$. The filter paper was transferred to sterile tubes for DNA extraction and subsequent PCR. The extracted DNA from the filter paper was used as quality control (QC). DNA integrity was analyzed by $1 \%$ agarose gel electrophoresis. DNA concentration and purity were verified using Nanodrop 2000 (Thermo). We used nested PCR to amplify the V-V4 region of bacterial 16s rRNA gene. During the first round, the 16s rRNA gene was amplified using the 27F (5'-AGA

Table 1 Clinical features of patients with thyroid carcinoma

\begin{tabular}{|c|c|c|c|c|c|c|c|c|c|}
\hline ID & Histology & Gender & Age & T stage & N stage & M stage & $\begin{array}{l}\text { Clinical } \\
\text { stage }\end{array}$ & $\begin{array}{l}\text { Extrathyroidal } \\
\text { extension }\end{array}$ & $\begin{array}{l}\text { Recurrence risk } \\
\text { stratification }\end{array}$ \\
\hline P01 & PTC & Male & 64 & $\mathrm{~T} 1 \mathrm{~b}$ & No & MO & 1 & Yes & Low risk \\
\hline P02 & PTC & Female & 47 & $\mathrm{~T} 1 \mathrm{~b}$ & NO & MO & 1 & No & Low risk \\
\hline P03 & PTC & Female & 49 & $\mathrm{~T} 1 \mathrm{a}$ & NO & MO & 1 & No & Low risk \\
\hline P04 & PTC & Male & 34 & $\mathrm{~T} 1 \mathrm{~b}$ & N1a & MO & 1 & No & Intermediate risk \\
\hline P05 & PTC & Female & 55 & T1a & No & MO & 1 & No & Low risk \\
\hline P06 & PTC & Female & 46 & $\mathrm{~T} 1 \mathrm{~b}$ & No & MO & 1 & No & Low risk \\
\hline P07 & PTC & Male & 55 & $\mathrm{~T} 1 \mathrm{a}$ & No & MO & I & No & Low risk \\
\hline P08 & PTC & Male & 21 & $\mathrm{~T} 1 \mathrm{~b}$ & N1a & MO & I & No & Intermediate risk \\
\hline P09 & PTC & Male & 53 & $\mathrm{~T} 1 \mathrm{a}$ & NO & MO & 1 & No & Low risk \\
\hline P10 & PTC & Male & 41 & $\mathrm{~T} 1 \mathrm{~b}$ & N1b & MO & 1 & No & Intermediate risk \\
\hline P11 & PTC & Male & 62 & $\mathrm{~T} 1 \mathrm{~b}$ & No & MO & । & No & Low risk \\
\hline P12 & PTC & Female & 59 & $\mathrm{~T} 2$ & N1 & MO & $\|$ & No & Low risk \\
\hline P13 & PTC & Female & 23 & T1b & No & MO & 1 & No & Low risk \\
\hline P14 & PTC & Female & 60 & $\mathrm{~T} 1 \mathrm{~b}$ & NO & MO & 1 & No & Low risk \\
\hline P15 & PTC & Female & 55 & T1a & N1a & MO & $\|$ & No & Low risk \\
\hline P16 & PTC & Female & 54 & T1a & No & MO & 1 & No & Low risk \\
\hline P17 & PTC & Female & 47 & $\mathrm{~T} 1 \mathrm{~b}$ & No & MO & 1 & No & Low risk \\
\hline P18 & PTC & Female & 40 & $\mathrm{~T} 1 \mathrm{~b}$ & N1a & MO & I & No & Intermediate risk \\
\hline P19 & PTC & Female & 39 & $\mathrm{~T} 1 \mathrm{~b}$ & N1a & MO & 1 & No & Intermediate risk \\
\hline P20 & PTC & Male & 29 & $\mathrm{~T} 1 \mathrm{~b}$ & $\mathrm{~N} 1 \mathrm{a}$ & MO & I & No & Intermediate risk \\
\hline P21 & PTC & Female & 50 & T1b & No & MO & 1 & No & Low risk \\
\hline P22 & PTC & Male & 34 & $\mathrm{~T} 1 \mathrm{~b}$ & N1a & MO & 1 & No & Intermediate risk \\
\hline P23 & PTC & Female & 55 & $\mathrm{~T} 1 \mathrm{~b}$ & N1 & MO & $\|$ & No & Intermediate risk \\
\hline P24 & PTC & Female & 52 & T1a & NO & MO & 1 & No & Low risk \\
\hline P25 & PTC & Female & 54 & $\mathrm{~T} 2$ & No & MO & 1 & Yes & Low risk \\
\hline P26 & PTC & Female & 25 & T1a & N1a & MO & 1 & No & Intermediate risk \\
\hline P27 & PTC & Female & 49 & $\mathrm{~T} 1 \mathrm{a}$ & NO & MO & I & No & Low risk \\
\hline P28 & PTC & Female & 47 & T1a & N1a & MO & I & No & Intermediate risk \\
\hline P29 & PTC & Female & 38 & $\mathrm{~T} 1 \mathrm{~b}$ & N1b & MO & 1 & Yes & Intermediate risk \\
\hline P30 & PTC & Female & 33 & $\mathrm{~T} 1 \mathrm{~b}$ & NO & MO & 1 & No & Low risk \\
\hline
\end{tabular}


GTTTGATCCTGGCTCAG-3') and 1492R (5'-GGTTAC CTTGTTACGACTT-3') primers. In the second round, V3-V4 region of 16S rRNA gene was amplified using the 341F (5'-TCGTCGGCAGCGT CAGATGTGTATAAGA GACAGCCTACGGGNGGCWGCAG-3') and 806R (5'-G TCTCGTGGGCTCGGAGATGTGTATAAGAGA CAGGACTACHVGGGTATCTAATCC-3') primers. All PCR reactions were carried out in $25 \mu \mathrm{L}$ reaction mixture containing $10 \mathrm{ng}$ of template DNA. Barcode was added using index PCR (Nextera XT Index Kit v2, illumina). The PCR condition of DNA from filter paper was the same as that of DNA from tissues. Index PCR products were sequenced with the Miseq platform.

\section{Sequencing data analysis}

The raw reads were filtered to obtain the high-quality clean reads using USEARCH 8.0. Chimera sequences were detected and removed using the UCHIME algorithm software [19]. Sequences with more than $97 \%$ similarity were allocated to one operational taxonomic unit (OTU) using UPARSE software [20]. The phylogenetic affiliation of each 16S rRNA gene sequence was analyzed by RDP Classifier against the Silva $16 \mathrm{~S}$ rRNA database using confidence threshold of $70 \%$. Subsequent analyses, including alpha diversity analysis, beta diversity analysis on Bray-Curtis distance, the linear discriminant analysis (LDA) effect size (LEfSe), and random forest analysis, were performed using MicrobiomeAnalyst [21].

\section{Statistical analysis}

The statistical Analyses were performed using GraphPad Prism (Version 8.0; GraphPad Software) software. Statistical significance was defined as a two-sided $P$-value of $<0.05$. The Mann-Whitney $U$ test was used to calculate the difference in Chao1 index, Shannon index, and the abundance of taxa between two groups.

\section{Results}

\section{Differences in microbial diversity and composition} between TC peritumor and tumor tissues

As shown in Additional file 1: Table S1, the 16s rRNA gene sequencing produced a median of 41,778 reads for QC samples, 30 paired tumor and peritumor tissues. First, we analyzed alpha diversity to investigate microbial diversity between TC peritumor and tumor tissues. The Chaol index reflecting species richness was lower in tumor tissues than in peritumor tissues; however, the difference was not significant $(P=0.268$, Fig. 2A). The Shannon index, which measures species richness and evenness, was significantly lower in tumor tissues in comparison to peritumor tissues $(P<0.001$, Fig. $2 \mathrm{~B})$. To exclude the possibility of contamination from the environment, six QC samples were obtained from the operating room where the tissue samples were collected. The analysis of alpha diversity showed that the Chao1 index and the Shannon index were both significantly lower in QC group compared with peritumor and tumor tissues $(P<0.001$, Fig. $2 \mathrm{~A}$ and $\mathrm{B})$.

To compare the composition of the microbial community between peritumor and tumor tissues, beta diversity was analyzed using Bray-Curtis method, and Principal coordinate analysis (PCoA) was performed, which showed that significant clustering was detected between QC and tissues samples. The PCoA also indicated that peritumor and tumor tissues showed two distinct clusters (PERMANOVA, $R^{2}=0.330, P<0.001$, Fig. 2 C).

The taxonomic profiles of thyroid microbiota are shown in Fig. 2D and E. We defined the core microbiota of thyroid tissues if it is observed in $80 \%$ of samples. At the phylum level, the core microbiota of thyroid was Proteobacteria, Bacteroidetes, Firmicutes (Fig. 2D, Additional file 2: Table S2 and Additional file 3: Table S3). At the genus level, the core microbiota of thyroid included Comamonas, Acinetobacter, Chryseobacterium, Pseudomonas, Microvirgula, Soonwooa, Sphingomonas (Fig. 2E, Additional file 4: Table S4 and Additional file 5: Table S5). The proportions of Comamonas, Acinetobacter, Microvirgula, and Soonwooa were lower in tumor tissues than in peritumor tissues (Fig. 2E). The tumor tissues had higher abundance of Sphingomonas compared with peritumor tissues (Fig. 2E). At the genus level, the core microbiota of QC samples included Enterobacter, Citrobacter, and Chryseobacterium, which were very different from those of thyroid (Fig. 2E).

To exclude the effect of environment on thyroid microbiota, we eliminated OTUs annotated as Enterobacter, Citrobacter, and Chryseobacterium from TC peritumor and tumor tissues. The Chaol index was higher in tumor tissues than in peritumor tissues; however, the difference was not significant $(P=0.224$, Fig. $3 \mathrm{~A})$. The Shannon index was significantly lower in tumor tissues than in peritumor tissues $(P=0.022$, Fig. 3B). PCoA showed that significant clustering was detected between tumor and peritumor tissues (PERMANOVA, $R^{2}=0.162, P<0.001$, Fig. 3C). At the phylum level, the core microbiota of thyroid was Proteobacteria, Bacteroidetes, Firmicutes (Fig. 3D). At the genus level, the core microbiota of thyroid included Sphingomonas, Comamonas, Acinetobacter, Pseudomonas, Microvirgula, and Soonwooa (Fig. 3E).

\section{Determination of differential taxa between different thyroid microhabitats}

To identify discriminative taxa between TC peritumor and tumor tissues, we analyzed the compositions of thyroid microbiota in peritumor and tumor tissues using linear discriminant analysis (LDA) effect size (LEfSe) 

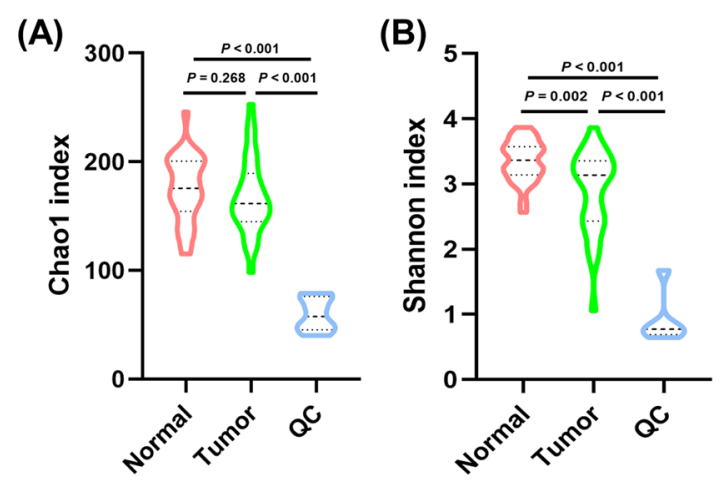

(C)

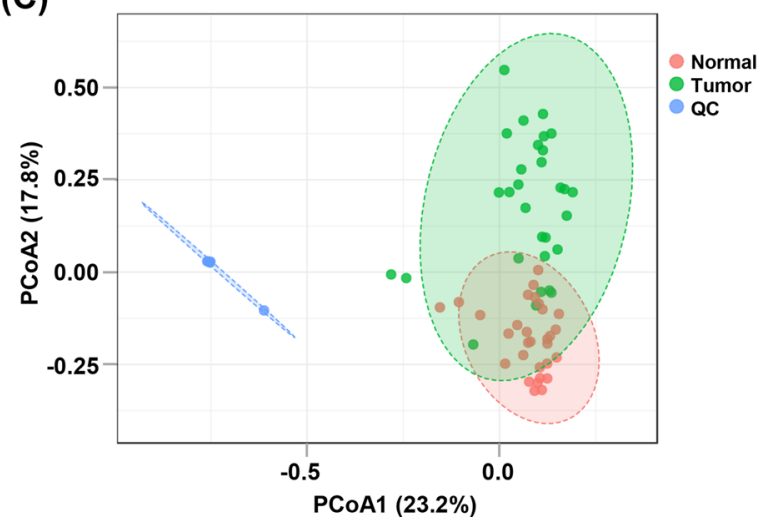

(D)

(E)
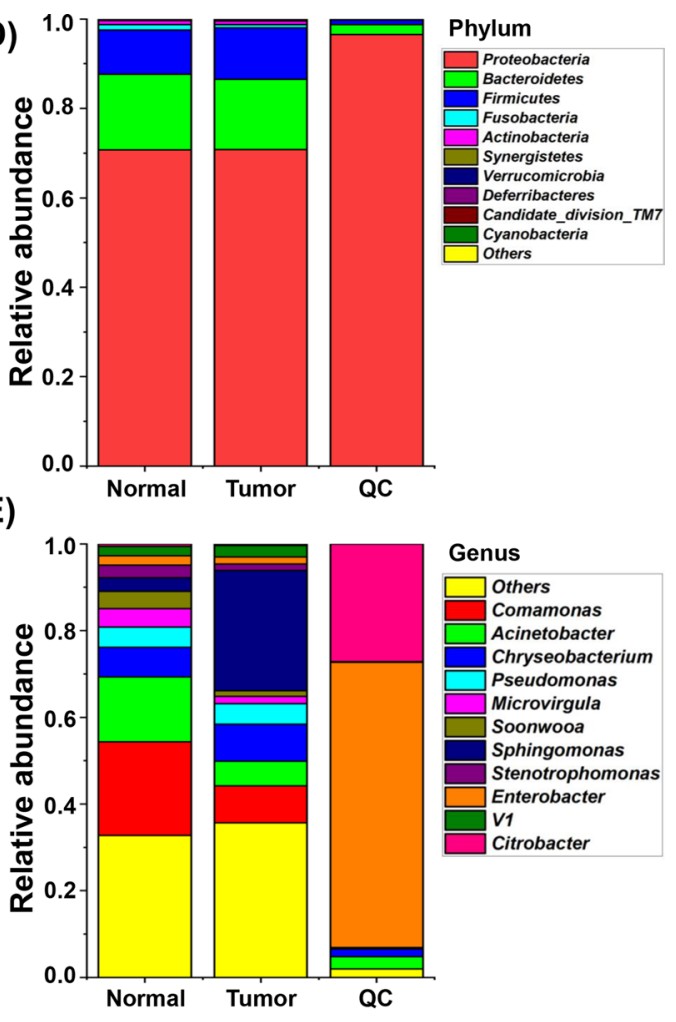

Fig. 2 Comparison of microbial profiles between QC samples, TC tumor and matched peritumor tissues before elimination of environmental contamination. A, B Chaol and Shannon indices were used to evaluate the microbial diversity of the paired tumor, peritumor tissues, and QC samples. Tumor and matched peritumor tissues were from 30 TC patients. QC samples were used to reveal the environmental microbiota. The Mann-Whitney $U$ test was performed to compare differences between two groups. C Principal coordinate analysis (PCoA) of Bray-Curtis analysis demonstrated that QC samples, peritumor and tumor tissues showed three distinct clusters. The microbial relative abundance of TC tumor tissues, matched peritumor tissues, and QC samples at the phylum (D) and genus (E) levels is shown

method (LDA $>3.0$, corrected $P$ value $<0.05)$. At the phylum level, we did not identify any differential taxa. At the genus level, the abundance of Sphingomonas and Aeromonas was significantly increased in peritumor tissues, whereas the abundance of Comamonas, Acinetobacter, Peptostreptococcus, and Proteus was significantly increased in tumor tissues (Fig. 4A). The 5 differential taxa including Sphingomonas, Aeromonas Comamonas, Acinetobacter and Peptostreptococcus were also confirmed by the random forest analysis which revealed 10 differential taxa between peritumor and tumor tissues (Fig. 4B). We further analyzed the differential abundance of the 5 discriminative features at the genus level between the two groups using the Mann-Whitney U test, and demonstrated that the abundance of these taxa was significantly different between the two groups $(P<0.05$, Fig. $4 \mathrm{C}-\mathrm{G})$.

\section{Identification of different thyroid microhabitats based on thyroid microbiota}

Receiver operating characteristic (ROC) curve analysis was performed to evaluate the diagnostic value of the 5 differential taxa in distinguishing tumor samples from peritumor samples. The areas under the curve (AUCs) of the 5 taxa ranged from 0.746 to 0.884 (Fig. 5). Comamonas and Sphingomonas had an AUC value $>0.800$ (Fig. $5 \mathrm{C}$ and $5 \mathrm{E}$ ), and were further selected as the potential biomarkers. The combination of Comamonas and Sphingomonas could rapidly increase the diagnostic accuracy in discriminating tumor samples from peritumor samples with an AUC value of 0.981 (95\% confidence interval [CI] 0.9491.000, Fig. 5F). 

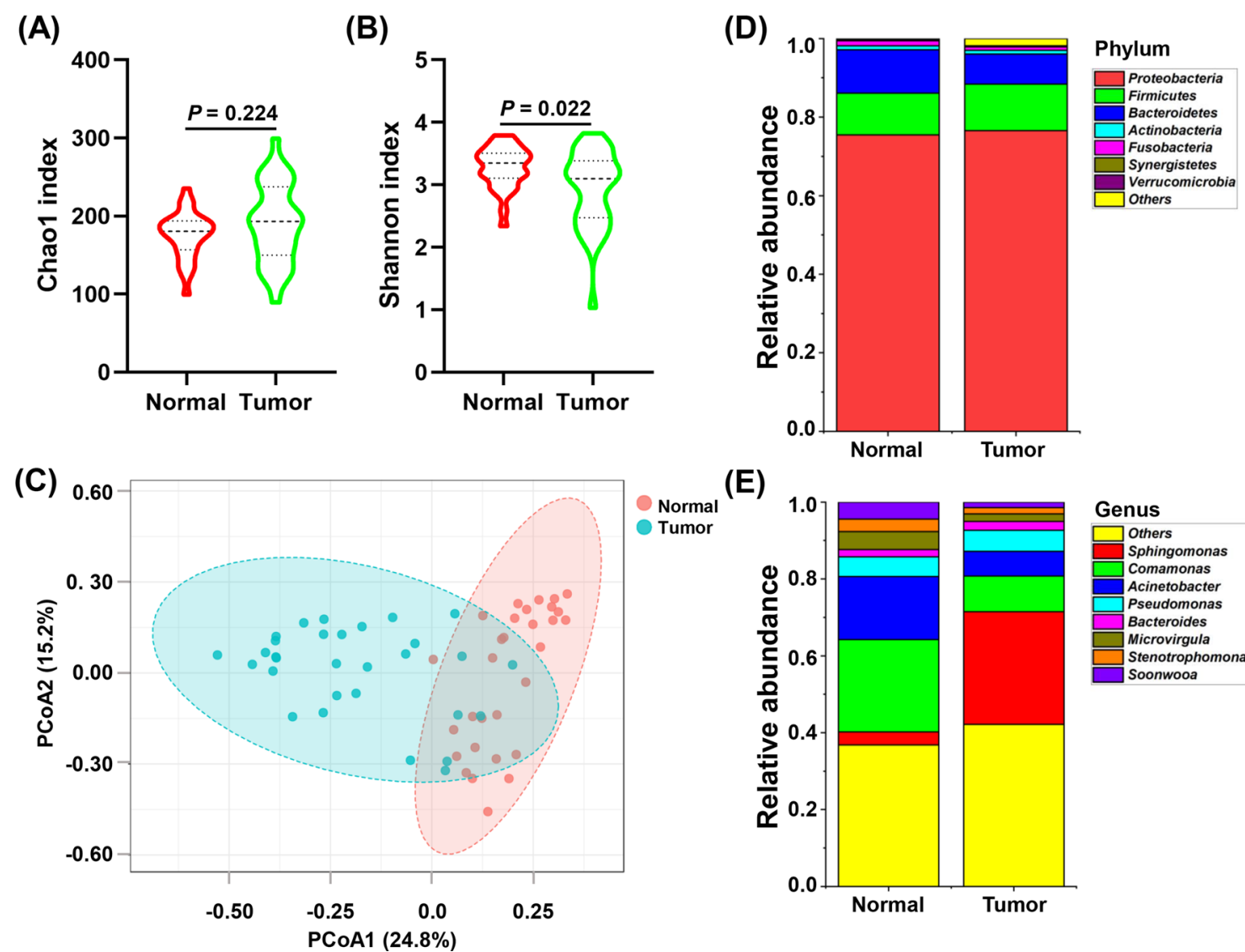

(E)

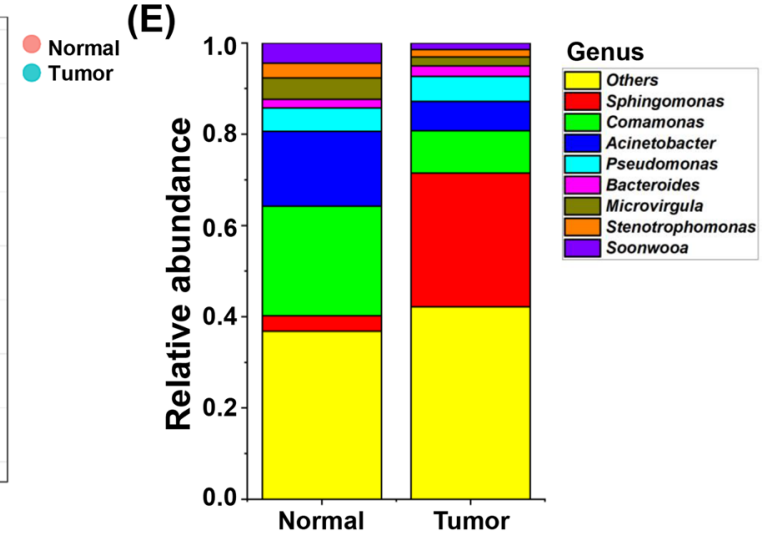

Fig. 3 Comparison of microbial profiles between TC tumor and matched peritumor tissues after elimination of environmental contamination. A, B Comparison of Chao 1 and Shannon indices between 30 TC tumor and matched peritumor tissues after elimination of environmental contamination. C Principal coordinate analysis (PCOA) demonstrated that the peritumor and tumor tissues showed two distinct clusters. The microbial relative abundance of 30 TC tumor tissues and matched peritumor tissues at the phylum (D) and genus (E) levels is shown

\section{The association between thyroid microbiota} and clinicopathological factors

To reveal the association between lymph node metastasis and thyroid microbiome, we analyzed microbial differences between tumor tissues from patients at N0 and N1 stage. The Chao1 index was significantly higher in N1 stage than in N0 stage $(P=0.049$, Fig. 6A), while the Shannon index was significantly lower in N1 stage in comparison to N0 stage $(P=0.020$, Fig. $6 \mathrm{~B})$. PCoA analysis based on the Bray-Curtis method showed that significant clustering was detected between patients at N0 and N1 stage (PERMANOVA, $\mathrm{R}^{2}=0.164, P<0.001$, Fig. $6 \mathrm{C}$ ). The LEfSe analysis showed that the abundance of Sphingomonas was significantly increased in N1 stage compared to N0 stage (Fig. 6D), which was also confirmed by the Mann-Whitney $U$ test $(P<0.001$, Fig. 6E). ROC curve analysis showed that Sphingomonas could distinguish between patients at $\mathrm{N} 0$ and N1 stage with an AUC of 0.964 (95\% CI 0.907-1.000, Fig. 6F). However, we observed no significant differences in Chao1 and Shannon indices between male and female patients $(P=0.349$, Fig. 6G; $P=0.657$, Fig. $6 \mathrm{H})$. PCoA analysis suggested that there was no significant difference in composition of thyroid microbiota between male and female patients (PERMANOVA, $\mathrm{R}^{2}=0.033, P=0.473$, Fig. 6I). LEfSe analysis revealed no differential taxa between male and female patients. Figure 6J-L showed that there was no difference in the diversity and composition of thyroid microbiota between patients aged $\geq 55$ and $<55$.

\section{Discussion}

In this study, we used 16s rRNA gene sequencing to characterize thyroid microbiota in different thyroid microhabitats. The alpha diversity and beta diversity were both significantly different between QC samples and thyroid tissue samples, indicating that thyroid tissues were not contaminated by the surrounding environment. We found that TC tumor tissues had lower thyroid microbiota richness and diversity than matched peritumor tissues. A decrease in microbiota diversity in tumor 


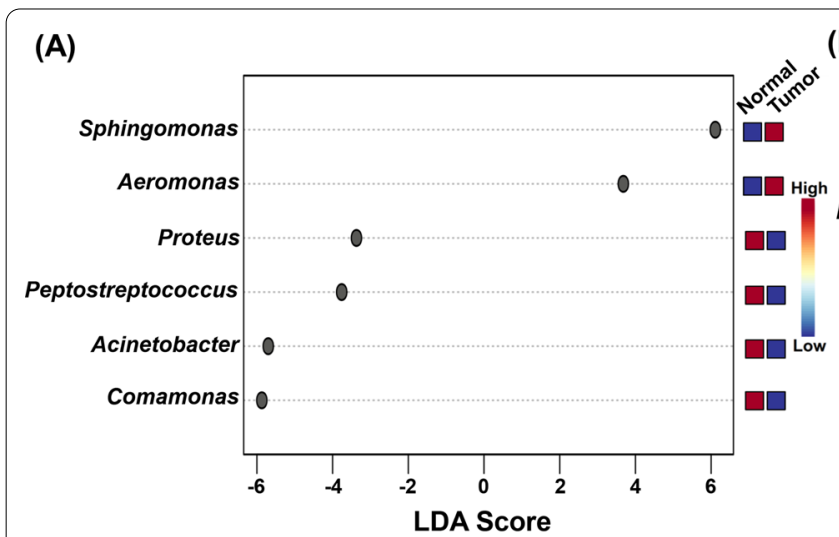

(C)

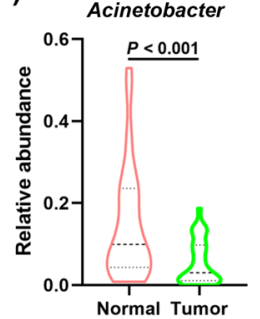

(D)

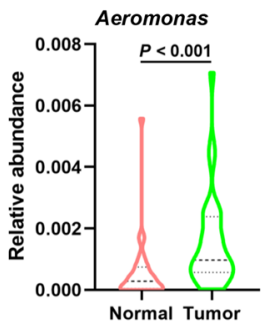

(E)

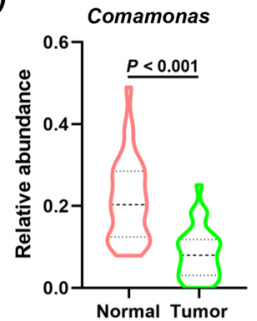

(B)

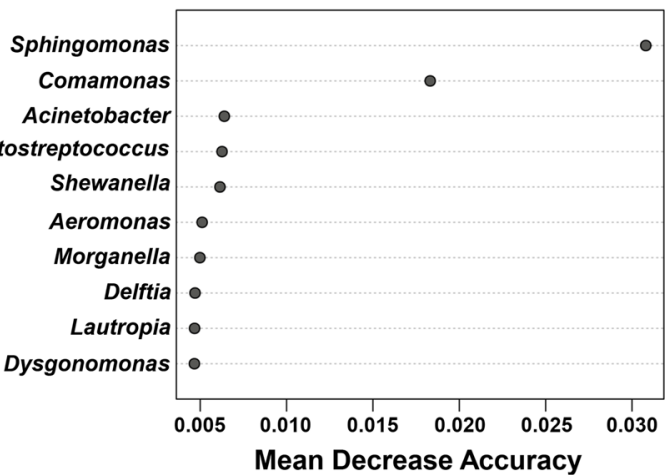

(F)

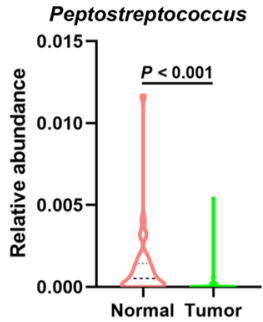

(G)

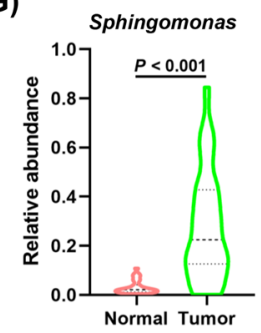

Fig. 4 The differential taxa at the genus level between the paired thyroid cancer tissues and peritumor tissues from 30 patients with thyroid cancer. A Differential taxa at the genus level identified by linear discriminant analysis (LDA) effect size (LEfSe) analysis (LDA $>3.0$, corrected $P$ value $<0.05$ ). B Differential taxa at the genus level identified by the random forest analysis. $\mathbf{C}-\mathbf{G}$ The differential abundance of the 5 discriminative genera between 30 thyroid cancer tissues and matched peritumor tissues was further validated using the Mann-Whitney $U$ test

(A)

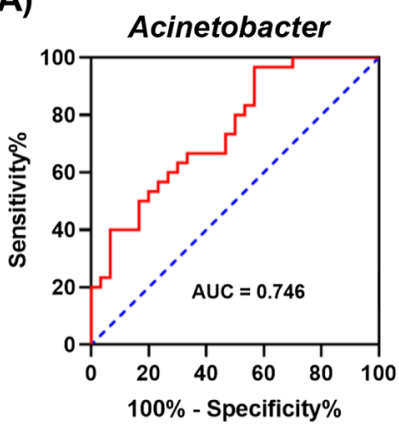

(D)

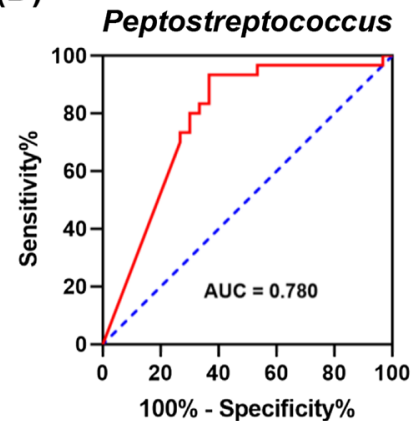

(B)

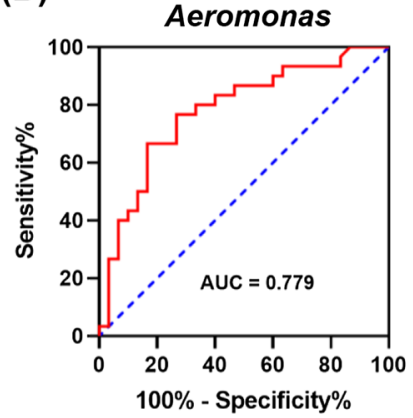

(E)

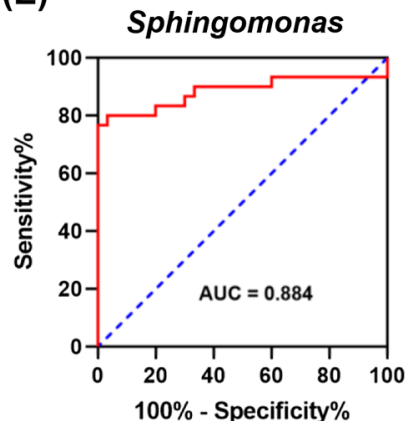

(C)

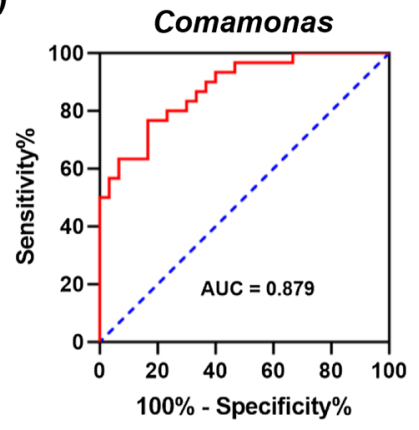

(F)
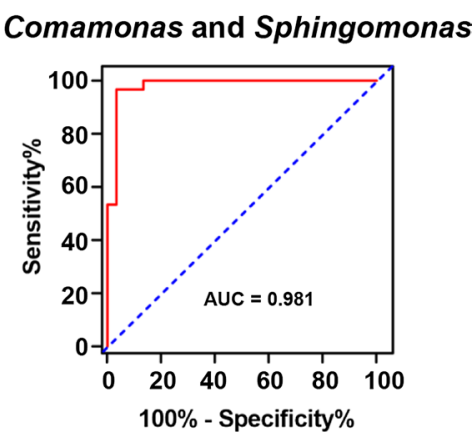

Fig. 5 Receiver operating characteristic (ROC) curves for differential genera. A-E ROC curves for the 5 differential genera and $\mathbf{F}$ the combination of Comamonas and Sphingomonas were plotted based on microbial relative abundance 
tissues was also observed in patients with lung cancer and gastric cancer [22, 23]. Nevertheless, an increase in gut microbiota diversity is observed in patients with TC, Hashimoto's thyroiditis, and hyperthyroidism [14, 16, 24]. At the genus level, the core microbiota of thyroid tissues included Comamonas, Acinetobacter, Pseudomonas, Microvirgula, Soonwooa, and Sphingomonas, while the core gut microbiota of TC encompassed Faecalibacterium, Bacteroides, Blautia, Rosebulia, Dialister, and Lachnoclostridium. These results showed that the composition of thyroid microbiota and gut microbiota of TC patients were different.

We identified 5 differential taxa, including the genus Sphingomonas and Aeromonas enriched in peritumor tissues, and Comamonas, Acinetobacter and Peptostreptococcus enriched in tumor tissues. However, the comparison of the gut microbial compositions between TC and healthy subjects showed that 27 genera, including Bacteroides, Roseburia, Megamonas, Klebsiella, Blautia, etc., markedly differed between the two groups with significantly differential abundance. These results demonstrated that the differential taxa of thyroid microbiota and gut microbiota were quite different, indicating different roles of thyroid microbiota and gut microbiota in promoting TC development.

More importantly, the higher abundance of Sphingomonas was associated with lymph node metastasis. In addition, patients enrolled in this study were at early stage (stage I and II). These results indicated that the abundance of Sphingomonas may represent a prognostic value for TC patients at early stage, and Sphingomonas may play a role in promoting TC development. An analysis of the global mucosa-associated microbiota revealed that the abundance of Sphingomonas was found to be increased in patients suffering colitis associated cancer compared with those with sporadic cancer [25]. Jeong et al. reported that the higher level of Comamonas in tumor tissues was associated with more metastasized lymph nodes in pancreatic cancer [26]. Comamonas is a cellulolytic microbe that could impact the host metabolism in cancer patients and play a role in inflammation [27]. Ling et al. reported that Comamonas was negatively associated with BDCA2 + pDCs, indicating its correlation with antitumor immunity [28]. These studies showed that Comamonas and Sphingomonas may play important roles in tumor progression. Furthermore, we found that the combination of Comamonas and Sphingomonas could discriminate tumor samples from peritumor samples with an AUC value of 0.981, indicating that the combination may be a powerful biomarker for TC.

A metagenomic analysis of the stool samples showed that the abundance of Acinetobacter was decreased in patients with colorectal cancer compared with healthy subjects [29]. A 16s rRNA gene sequencing analysis of lung tissues revealed that the microbial community compositions of patients only with emphysema were characterized by a significantly higher abundance of Proteobacteria (primary the genus Acinetobacter) in comparison to lung cancer patients with or without emphysema [23]. Acinetobacter is widespread in natural environments and plays an important role in disseminating infections, including the respiratory tract and urinary tract. Certain species of Acinetobacter are resistant to multi-drugs and regarded as important pathogens. Acinetobacter baumannii is an important opportunistic pathogen that is ubiquitous in hospitals and other settings related with healthcare. A systematic review of thirteen original articles showed that gastric carcinogenesis could be associated with an increase in the abundance of Acinetobacter baumannii [30]. These studies showed that the genus Acinetobacter could be related with carcinogenesis of different malignancies. We found that the genus Acinetobacter was enriched in TC tumor tissues, indicating that it may promote TC progression.

Meanwhile, this study has a few limitations. First, the sample size in this study was small. This study has obtained several positive results, which pave the way for future study with larger sample size. The quantitative reverse-transcription polymerase chain reaction (RT-qPCR) assay is a common quantitative approach to identify microbes in tumor tissues. In the future, we will utilize RT-qPCR to quantitatively identify bacteria, particularly Comamonas and Sphingomonas, in thyroid cancer tissues in larger sample size. Second, this was a

\footnotetext{
(See figure on next page.)

Fig. 6 The association between clinicopathological factors and thyroid microbiota. A, B The differences in Chao1 and Shannon indices between tumor tissues from thyroid cancer patients at N0 and N1 stage. Mann-Whitney U tests were performed. C Principal coordinate analysis (PCoA) based on Bray-Curtis distance revealed that thyroid cancer patients at N0 stage were significantly different from those at N1 stage. D Linear discriminant analysis (LDA) effect size (LEfSe) analysis (LDA>3.0, corrected $P$ value $<0.05$ ) was performed to evaluate differential taxa at the genus level. E The differential abundance of Sphingomonas between NO and N1 stage was further validated using the Mann-Whitney $\mathrm{U}$ test. F The receiver operating characteristic (ROC) curve for Sphingomonas genera was plotted using microbial relative abundance to assess the value of thyroid microbiota as a diagnostic tool to distinguish between thyroid patients with NO and N1 stage. G, H The differences in Chao1 and Shannon indices between tumor tissues from male and female patients with thyroid cancer. Mann-Whitney $U$ tests were performed. I Principal coordinate analysis (PCoA) of thyroid microbiota in male and female patients with thyroid cancer based on Bray-Curtis distance. J, K The differences in Chaol and Shannon indices between tumor tissues from thyroid cancer patients aged $\geq 55$ and $<55$. Mann-Whitney $U$ tests were performed. $\mathbf{L}$ Principal coordinate analysis (PCOA) of thyroid microbiota in patients with thyroid cancer aged $\geq 55$ and $<55$ based on Bray-Curtis distance
} 
(A)

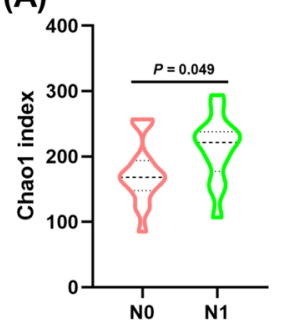

(C)

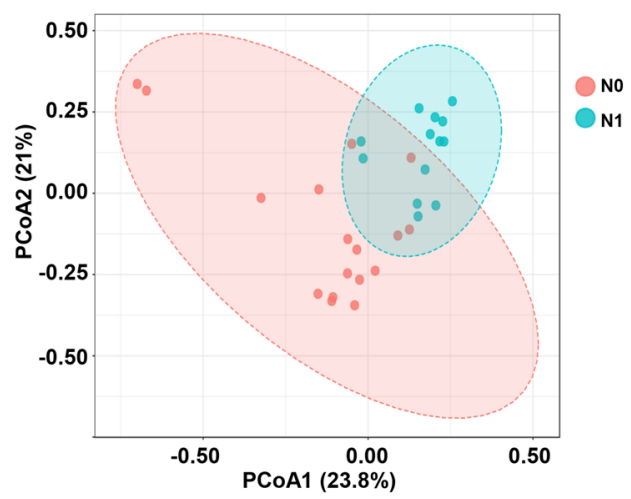

(G)

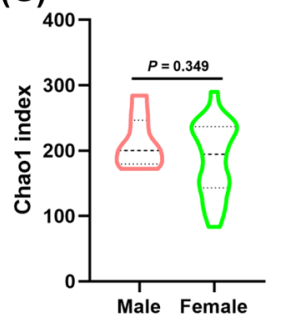

(l)
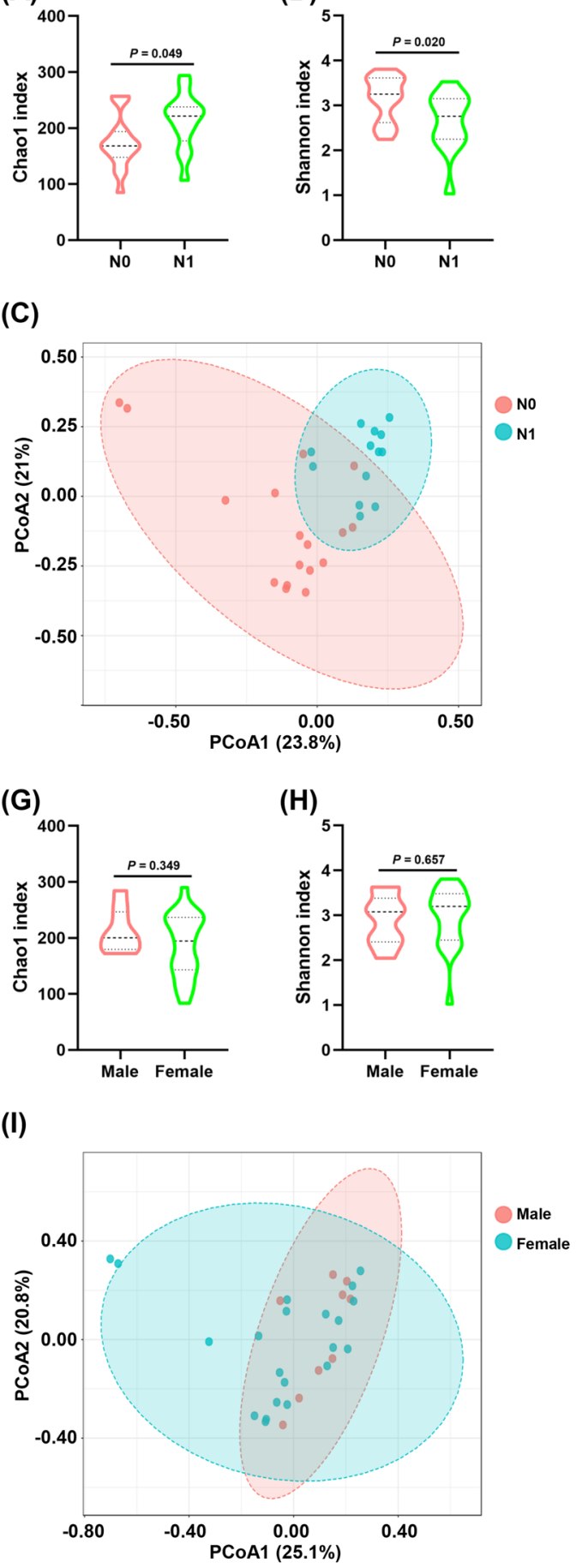

(D)

(E)

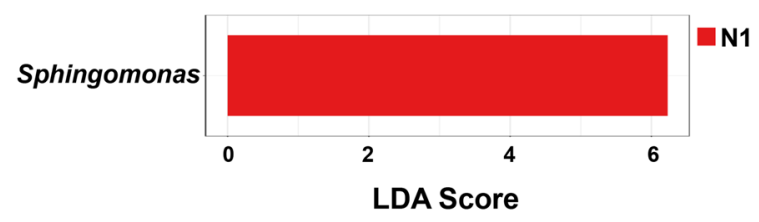

(F)
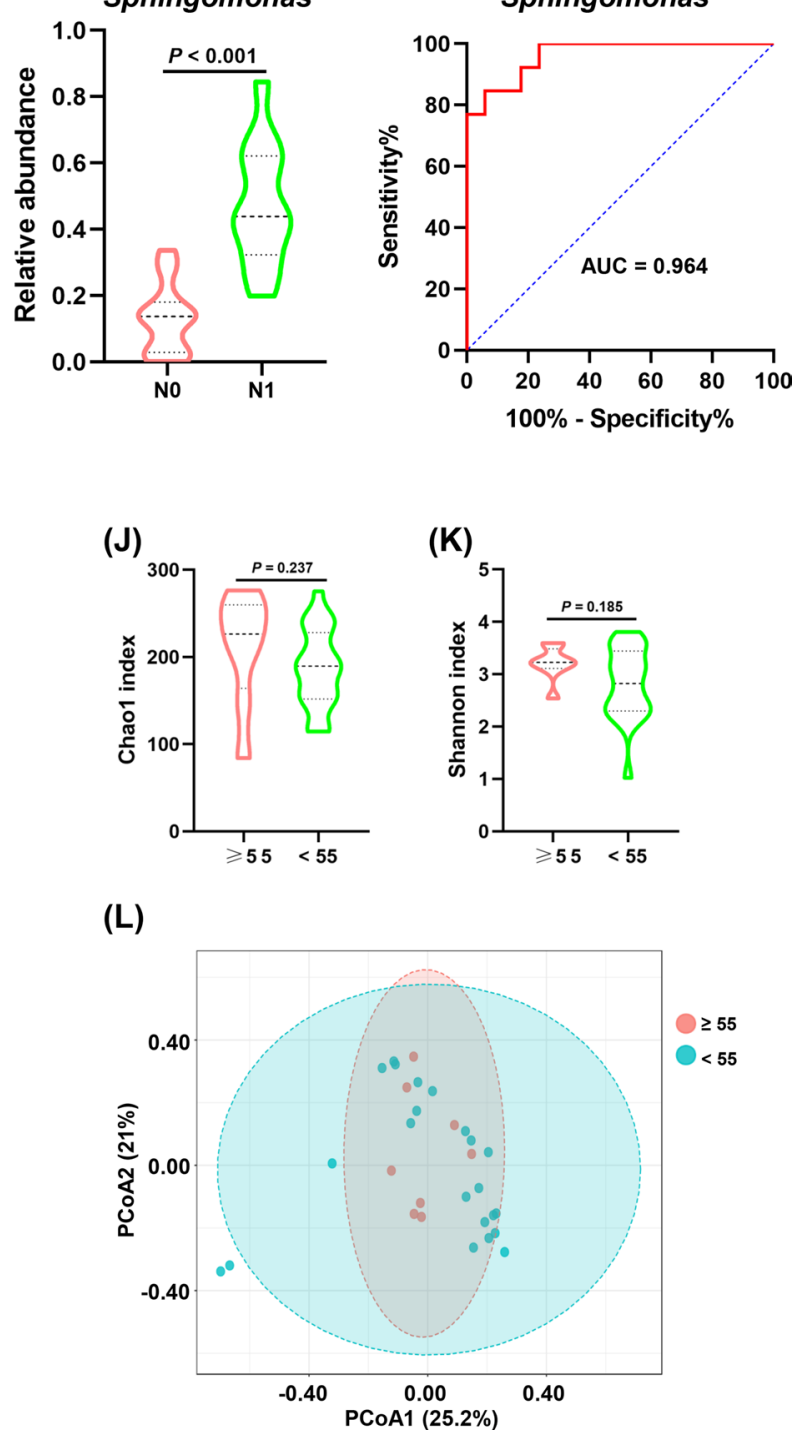

(L)

(J)

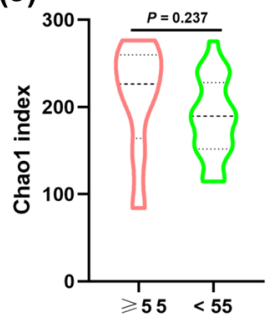

(K)

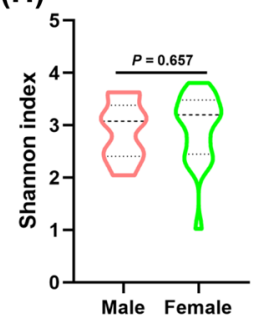

Female

Fig. 6 (See legend on previous page.)

retrospective study, and a prospective study is needed to validate our results. Third, the lack of a control group with benign nodules may compromise the interpretation of the results, since comparing the microbial difference of thyroid tissues between patients with benign nodules and TC will further support the observations of this work. In addition, 16s rRNA sequencing cannot identify the specific bacterial species, resulting in that information on the species of thyroid microbiota was 
not obtained. Thus, a study revealing the species of thyroid microbiota is needed in future.

\section{Conclusion}

Taken together, we found that the richness and diversity of thyroid microbiota were significantly lower in TC tumor samples in comparison with matched peritumor tissues. The abundance of genus Sphingomonas and Aeromonas was increased in tumor tissues, while the abundance of Comamonas, Acinetobacter, and Peptostreptococcus was enhanced in peritumor tissues. The combination of Sphingomonas and Comamonas could serve as a powerful marker for distinguishing TC tumor tissues from matched peritumor tissues. Furthermore, the increased abundance of Sphingomonas was correlated with lymph node metastasis, indicating that the abundance of Sphingomonas may represent a prognostic value for TC patients at early stage, and Sphingomonas may play a role in promoting TC development.

\section{Abbreviations}

ATC: Anaplastic thyroid carcinoma; AUC: Area under the curve; BMI: Body mass index; Cl: Confidence interval; FTC: Follicular thyroid carcinoma; HCTC: Hurthle cell thyroid carcinoma; LEfSe: Linear discriminant analysis (LDA) effect size; MTC: Medullary thyroid carcinoma; OTU: Operational taxonomic unit; PCoA: Principal coordinate analysis; PTC: Papillary thyroid carcinoma; QC: Quality control; ROC: Receiver operating characteristic; TC: Thyroid carcinoma.

\section{Supplementary Information}

The online version contains supplementary material available at https://doi. org/10.1186/s12967-021-03167-9.

Additional file 1: Table S1. Sequencing reads of samples by 16 s rRNA sequencing.

Additional file 2: Table S2. The identified phylum in each samples.

Additional file 3: Table S3. The identified phylum in each group.

Additional file 4: Table S4. The identified genera in each sample.

Additional file 5: Table S5. The identified genera in each group.

\section{Acknowledgements}

The authors would like to thank all patients who participated in this study.

\section{Authors' contributions}

DD: Conceptualization, methodology, software, validation, investigation, data curation, formal analysis, visualization, writing-original draft, writing-review \& editing, funding acquisition. Yan Y: Conceptualization, resources, investigation. Yong Y: Conceptualization, methodology, software, validation, investigation, data curation, formal analysis, visualization, writing-original draft, writingreview \& editing. TD: Investigation, data curation. Jiansheng X: Supervision. WW: Resources, project administration. LT: Conceptualization, resources, supervision, funding acquisition. Juan X: Supervision, funding acquisition. JY: Conceptualization, writing-review \& editing, supervision, funding acquisition. $\mathrm{HJ}$ : Conceptualization, writing-review \& editing, project administration, supervision. All authors read and approved the final manuscript.

\section{Funding}

This study was supported by the National Natural Science Foundation of China (Nos. 81860182, 81772853, and 82160527), the Natural Science Foundation of Jiangxi Province (No. 20181BAB205036), Project of the Regional
Diagnosis and Treatment Center of the Health Planning Committee (No. JBZX-201903), Science and Technology Department of Jiangxi Province (No. 20203BBGL73201), Health Commission of Jiangxi Province (Nos. 20203229, 202130016), and The First Affiliated Hospital of Nanchang University (No. YFYPY202002). Basic Public Welfare Research Project of Zhejiang Province (No. LGF2OH160023), and Medical Health Science and Technology Project of Zhejiang Province (No. 2018273130).

\section{Availability of data and materials}

The original data presented in the study are included in the article, further inquiries can be directed to the corresponding authors.

\section{Declarations}

\section{Ethics approval and consent to participate}

This study was conducted in accordance with the code of ethics of the World Medical Association (Declaration of Helsinki) and the studies involving human participants were reviewed and approved by the First Affiliated Hospital, School of Medicine, Zhejiang University, Hangzhou, China. The study was approved by the Medical Research Ethics Committee of the hospital (2020845). The patients provided their written informed consent to participate in this study.

\section{Consent for publication}

All authors give consent for publication.

\section{Competing interests}

The authors have no conflicts of interest to declare.

\section{Author details}

${ }^{1}$ Jiangxi Otorhinolaryngology Head and Neck Surgery Institute, Department of Otorhinolaryngology Head and Neck Surgery, The First Affiliated Hospital of Nanchang University, Nanchang, China. ${ }^{2}$ Department of Surgical Oncology, The First Affiliated Hospital, School of Medicine, Zhejiang University, Hangzhou, China. ${ }^{3}$ Department of Otolaryngology Head and Neck Surgery, Jiangxi Provincial People's Hospital Affiliated to Nanchang University, Nanchang, China. ${ }^{4}$ Department of General Surgery, The First Affiliated Hospital of Nanchang University, Nanchang, China. ${ }^{5}$ Pathology Department, Taizhou Hospital of Zhejiang Province Affiliated to Wenzhou Medical University, Linhai, China.

Received: 12 October 2021 Accepted: 23 November 2021

Published online: 30 November 2021

\section{References}

1. Bray F, Ferlay J, Soerjomataram I, Siegel RL, Torre LA, Jemal A. Global cancer statistics 2018: GLOBOCAN estimates of incidence and mortality worldwide for 36 cancers in 185 countries. CA Cancer J Clin. 2018;68:394-424.

2. Pellegriti G, Frasca F, Regalbuto C, Squatrito S, Vigneri R. Worldwide increasing incidence of thyroid cancer: update on epidemiology and risk factors. J Cancer Epidemiol. 2013;2013:965212.

3. Alfano M, Canducci F, Nebuloni M, Clementi M, Montorsi F, Salonia A. The interplay of extracellular matrix and microbiome in urothelial bladder cancer. Nat Rev Urol. 2016;13:77-90.

4. Liu HX, Tao LL, Zhang J, Zhu YG, Zheng Y, Liu D, et al. Difference of lower airway microbiome in bilateral protected specimen brush between lung cancer patients with unilateral lobar masses and control subjects. Int J Cancer. 2018;4:769-78.

5. Qin J, Li R, Raes J, Arumugam M, Burgdorf KS, Manichanh C, et al. A human gut microbial gene catalogue established by metagenomic sequencing. Nature. 2010;464:59-65.

6. lida N, Dzutsev A, Stewart CA, Smith L, Bouladoux N, Weingarten RA, et al. Commensal bacteria control cancer response to therapy by modulating the tumor microenvironment. Science. 2013;342:967-70.

7. Lee YK, Mazmanian SK. Has the microbiota played a critical role in the evolution of the adaptive immune system? Science. 2010;330:1768-73.

8. Schwabe RF, Jobin C. The microbiome and cancer. Nat Rev Cancer. 2013;13:800-12. 
9. Fodor AA, Klem ER, Gilpin DF, Elborn JS, Boucher RC, Tunney MM, et al. The adult cystic fibrosis airway microbiota is stable over time and infection type, and highly resilient to antibiotic treatment of exacerbations. PLOS ONE. 2012;7:e45001.

10. Huang YJ, Nelson CE, Brodie EL, Desantis TZ, Baek MS, Liu J, et al. Airway microbiota and bronchial hyperresponsiveness in patients with suboptimally controlled asthma. J Allergy Clin Immunol. 2011;127:372-81.

11. Sze MA, Dimitriu PA, Hayashi S, Elliott WM, McDonough JE, Gosselink $J$, et al. The lung tissue microbiome in chronic obstructive pulmonary disease. Am J Respir Crit Care Med. 2012;185:1073-80.

12. Hosgood HD, Sapkota AR, Rothman N, Rohan T, Hu W, Xu J, et al. The potential role of lung microbiota in lung cancer attributed to household coal burning exposures. Environ Mol Mutagen. 2014;55:643-51.

13. Ishaq HM, Mohammad IS, Shahzad M, Ma C, Raza MA, Wu X, et al. Molecular alteration analysis of human gut microbial composition in Graves' disease patients. Int J Biol Sci. 2018;14:1558-70.

14. Zhou L, Li X, Ahmed A, Wu D, Liu L, Qiu J, et al. Gut microbe analysis between hyperthyroid and healthy individuals. Curr Microbiol. 2014;69:675-80

15. Su X, Zhao Y, Li Y, Ma S, Wang Z. Gut dysbiosis is associated with primary hypothyroidism with interaction on gut-thyroid axis. Clin Sci. 2020;134:1521-35.

16. Feng J, Zhao F, Sun J, Lin B, Zhao L, Liu Y, et al. Alterations in the gut microbiota and metabolite profiles of thyroid carcinoma patients. Int J Cancer. 2019;2019(144):2728-45.

17. Tudhope GR, Wilson GM. Anaemia in hypothyroidism. Incidence, pathogenesis, and response to treatment. Q J Med. 1960;29:513-37.

18. Cellini M, Santaguida MG, Virili C, Capriello S, Brusca N, Gargano L, et al. Hashimoto's thyroiditis and autoimmune gastritis. Front Endocrinol. 2017:8:92.

19. Edgar RC, Haas BJ, Clemente JC, Quince C, Knight R. UCHIME improves sensitivity and speed of chimera detection. Bioinformatics. 2011;27:2194-200.

20. Edgar RC. UPARSE: highly accurate OTU sequences from microbial amplicon reads. Nat Methods. 2013;10:996-8.

21. Dhariwal A, Chong J, Habib S, King IL, Agellon LB, Xia J. MicrobiomeAnalyst: a web-based tool for comprehensive statistical, visual and metaanalysis of microbiome data. Nucleic Acids Res. 2017;45:W180-188.
22. Liu X, Shao L, Liu X, Ji F, Mei Y, Cheng Y, et al. Alterations of gastric mucosal microbiota across different stomach microhabitats in a cohort of 276 patients with gastric cancer. EBioMedicine. 2019;40:336-48.

23. Liu Y, O'Brien JL, Ajami NJ, Scheurer ME, Amirian ES, Armstrong G, et al. Lung tissue microbial profile in lung cancer is distinct from emphysema. Am J Cancer Res. 2018;8:1775-87.

24. Zhao F, Feng J, Li J, Zhao L, Liu Y, Chen $\mathrm{H}$, et al. Alterations of the gut microbiota in Hashimoto's thyroiditis patients. Thyroid. 2018;28:175-86.

25. Richard ML, Liguori G, Lamas B, Brandi G, Costa G, Hoffmann TW, et al. Mucosa-associated microbiota dysbiosis in colitis associated cancer. Gut Microbes. 2018;9:131-42.

26. Jeong JY, Kim TB, Kim J, Choi HW, Kim EJ, Yoo HJ, et al. Diversity in the extracellular vesicle-derived microbiome of tissues according to tumor progression in pancreatic cancer. Cancers. 2020;12:2346.

27. Kanokratana P, Wongwilaiwalin S, Mhuantong W, Tangphatsornruang S, Eurwilaichitr L, Champreda V. Characterization of cellulolytic microbial consortium enriched on Napier grass using metagenomic approaches. J Biosci Bioeng. 2018;125:439-47.

28. Ling Z, Shao L, Liu X, Cheng Y, Yan C, Mei Y, et al. Regulatory T cells and plasmacytoid dendritic cells within the tumor microenvironment in gastric cancer are correlated with gastric microbiota dysbiosis: a preliminary study. Front Immunol. 2019;10:533.

29. Yang J, McDowell A, Kim EK, Seo H, Lee WH, Moon CM, et al. Development of a colorectal cancer diagnostic model and dietary risk assessment through gut microbiome analysis. Exp Mol Med. 2019;51:1-15.

30. Dias-Jácome E, Libânio D, Borges-Canha M, Galaghar A, PimentelNunes P. Gastric microbiota and carcinogenesis: the role of nonHelicobacter pylori bacteria—a systematic review. Rev Esp Enferm Dig. 2016;108:530-40.

\section{Publisher's Note}

Springer Nature remains neutral with regard to jurisdictional claims in published maps and institutional affiliations.

Ready to submit your research? Choose BMC and benefit from:

- fast, convenient online submission

- thorough peer review by experienced researchers in your field

- rapid publication on acceptance

- support for research data, including large and complex data types

- gold Open Access which fosters wider collaboration and increased citations

- maximum visibility for your research: over $100 \mathrm{M}$ website views per year

At BMC, research is always in progress.

Learn more biomedcentral.com/submissions 